\title{
The Role of Antioxidants in the Covid-19 Pandemic
}

\author{
Demir $\mathbf{H}^{1}$ and Kaçmaz $\mathbf{R}^{2 *}$ \\ ${ }^{1}$ Van Yüzüncü Yil Üniversitesi, Fen Fakültesi Kimya Bölümü, Biyokimya ABD, Turkey \\ ${ }^{2}$ Van İl Sağlik Müdürlüğü, Sağlik Hizmetleri Başkanliği, Turkey
}

*Corresponding author: R Raci Kaçmaz, Van Yüzüncü Yil Üniversitesi, Cevdetpaşa Mah. Emniyet Cad. 1 No:54 A Yağmur Sitesi A/Blok No:10 İpekyolu/VAN, Turkey, Tel: 05053735855;

\section{Review article}

Volume 5 Issue 3

Received Date: December 19, 2020

Published Date: December 30, 2020

DOI: $10.23880 /$ ijbp-16000190 Email: kacmazraci@hotmail.com

\section{Abstract}

Covid-19 disease caused by Severe Acute Respiratory Syndrome Coronavirus 2 (SARS-CoV-2), which has spread rapidly in many countries, was declared as a pandemic by the World Health Organization (WHO) on March 11, 2020. Covid-19 disease poses a threat to the health systems and economies of countries. Covid-19 is taken into the cell by endocytosis by binding to Angiotensin Converting Enzyme 2 (Angiotensin-Con-verting Enzyme 2, ACE 2), which is found in alveolar macrophages, monocytes and epithelial cells in the respiratory system as a host cell receptor. Here the replicated virus is released intracellularly and infects other target cells. Studies have reported that the cytokine storm that causes the clinical course of Covid-19 patients to deteriorate can be relieved with antioxidant agents. Since there is no drug or other therapeutic substance currently approved for the treatment of Covid-19 disease, keeping the immune system active and strong is vital in terms of preventing and managing the disease. The most important factor in strengthening immunity and protecting health; It is an optimal diet that is adequate and balanced in terms of macronutrients and rich in micronutrients. Experimental studies have shown that antioxidant vitamins (A, C and E), omega-3 fatty acids, pre / probiotics and trace elements (zinc, selenium) play key roles in supporting the human immune system and reducing the risk of infection. In this study, the role of antioxidant substances known to have an important role in the immune system in terms of prevention and treatment of Covid-19 infection was examined.

Keywords: Covid 19; Immune system; Antioxidant; Vitamins; Minerals

\section{Introduction}

Covid-19 disease, which was declared as a pandemic by the World Health Organization (WHO) on March 11, 2020; Severe acute respiratory syndrome is caused by coronavirus 2 (SARS-CoV-2) [1,2]. Coronavirus is an enveloped RNA virus that can cause a variety of symptoms such as pneumonia, fever, difficulty breathing, and cough. A coronavirus; The nucleocapsid consists of four structural proteins: envelope, membrane, and rod-like protrusions (spines). Since these protrusions are called "corona", which means crown in Latin, these viruses are called coronavirus (crowned virus). Coronavirus belongs to the Coronaviridae family and according to its genotypic and serological characteristics; It is divided into four different subgroups as alpha, beta, gamma and delta. While it can infect humans with alpha and beta coronaviruses, it can infect animals with gamma and delta coronaviruses $[3,4]$.

Covid-19; rapidly spreading in many countries, together with the high rate of hospitalizations requiring intensive care, a global health problem that can quickly cause Acute Respiratory Distress Syndrome (ARDS), respiratory failure, multi-organ failure and deaths of up to approximately ( 10\%). transformed. Covid-19 disease has become an important threat to the health systems and economies of 


\section{International Journal of Biochemistry \& Physiology}

countries.

Covid-19 is taken into the cell by endocytosis by binding to Angiotensin Converting Enzyme 2 (AngiotensinCon-verting Enzyme 2, ACE 2), which is found in alveolar macrophage, monocyte and epithelial cells in the respiratory system as a host cell receptor is released and infects other target cells [5]. Virus-induced cytokine storm caused by cytokines such as IL-2, IL-6, IL-7, TNF-a may be the main cause of organ damage. Later, pulmonary edema, respiratory distress, acute respiratory distress syndrome (ARDS), acute cardiac damage and secondary infection that can lead to death may develop [6].

Studies have reported that the storm of cytokines (polypeptide molecules secreted in response to microbes and other antigens, regulating immune and inflammatory reactions) that cause deterioration of the clinical course in Covid-19 patients can be relieved by antioxidant agents $[7,8]$. In some studies conducted in the light of this information, it has been stated that nutrients with immune modulator and antioxidant properties can be effective in the treatment of Covid-19 [9]. Currently, there is no drug that can definitively prevent or treat the Covid-19 epidemic, so keeping the immune system active and strong is important for preventing and managing the disease [2,10-12].

In this article, antioxidant substances known to have an important role in the immune system in terms of prevention and treatment of Covid-19 infection were examined.

\section{Oxidative Stress and Antioxidant Defense}

In biological systems, oxidative stress is a complex process characterized by an imbalance between the production of free radicals and the body's destruction of these reactive species using endogenous and exogenous antioxidants (defense systems and compounds that prevent, slow or stop oxidative damage by neutralizing free radicals) [13-15].

Free radicals are atoms or molecules that contain one or more unpaired electrons in their outermost orbit [16]. Free radicals can be produced endogenously and exogenously. Endogenous sources are mitochondrial, microsomal and membrane electron transport chain, phagocytic cells, endothelial cells, and oxidant enzymes [13-15]. Ultraviolet rays, cigarette smoke, alcohol, chemicals such as cleaning products, pesticides, burning organic materials during cooking, air pollutants such as carbon monoxide are exogenous sources that cause free radical production $[13,15,17]$. The amount of free radicals increases during many diseases in the organism such as cancer and cardiovascular diseases $[13,18]$.
In biological systems, there are enzymatic and nonenzymatic antioxidant mechanisms that enable free radicals to be neutralized $[13,15]$. When the organism is exposed to excessive reactive oxygen species (Superoxide $\left(02^{\cdot-}\right)$, hydroxyl $\left(\mathrm{OH}^{\circ}\right)$ and hydrogen peroxide $\left(\mathrm{H}_{2} \mathrm{O}_{2}\right)$ ) The antioxidant system (enzymes such as superoxide dismutase, catalase, glutathione peroxidase, bilirubin and albumin) is compromised and consequently the organism cannot be fully protected. The body uses exogenous antioxidants (Vitamin A, Vitamin C, Vitamin E etc.) supplied through food, nutritional supplements to compensate for this antioxidant deficit [19]. Therefore, antioxidant vitamins are vitamins that prevent oxidation and strengthen the immune system [15].

Antioxidant vitamins; They act by using at least one of the 6 mechanisms in providing oxidant-antioxidant balance. collecting free radicals, suppressing their reactions and breaking the reaction chain, repairing protein, lipid and DNA molecules, preventing cellular kinase losses, and increasing the synthesis of antioxidant enzymes $[13,15,19]$.

\section{Antioxidants}

\section{Vitamin A}

Vitamin A participates in many physiological processes, such as maintaining vision, reproduction and brain function, supporting growth and development, ensuring epithelial and mucus integrity, and cell differentiation $[13,20]$. In some studies, it has been emphasized that vitamin A may be a promising alternative in the treatment of Covid-19 outbreak and prevention of lung infection [21]. It has been reported that the immune system is suppressed in individuals with vitamin A deficiency and individuals become more susceptible to infections, and vitamin A has even been described as an "anti-infective" vitamin for many years [22]. Vitamin A deficiency has been associated with an increased risk of infection [23]. Vitamin A plays a regulatory role in cellular immunity and humoral immunity (antibody formation) responses [2]. Vitamin A supplementation has been reported to increase humoral immunity following influenza vaccination of pediatric patients [13]. In the study, it was reported that as a result of the modulation of immune system function by vitamin A, mortality (mortality rate) and morbidity (morbidity rate) decreased depending on the type of infection in viral infections [23]. It has been stated that vitamin A plays an important role in the differentiation, maturation and regulation of the cells in the innate immune system. Vitamin A improves the phagocytic capacity of macrophages, natural killer [24]. In individuals with low vitamin A levels, histopathological changes occur in the lung epithelium and lung parenchyma (the whole lung), resulting in an increased risk of lung dysfunction and respiratory disease. This is particularly important when considering the negative effects of Covid-19 on lung function [13,25]. 


\section{International Journal of Biochemistry \& Physiology}

\section{Vitamin E}

Vitamin E is a lipid-soluble, natural antioxidant that is thought to support endogenous defenses against oxidative stress caused by increased reactive oxygen species (ROS) formation [26]. Vitamin E has an immunomodulatory effect with its antioxidant activity [27]. It has been reported that vitamin E deficiency causes impairment of both humoral and cellular immunity $[2,28]$. E vitamin has been reported to protect cell membrane integrity against free radical damage $[9,29]$. In some studies, it has been stated that vitamin E can play a protective role against Covid-19 [30]. It has been reported that vitamin $\mathrm{E}$, with its powerful antioxidant properties, may play a role in preventing COVID-19 induced ARDS induction and reducing disease severity [24].

\section{Vitamin C}

Vitamin C (ascorbic acid) is an antioxidant and enzymatic cofactor required for many physiological reactions, ranging from preventing viral infections, providing collagen biosynthesis, and preserving epithelial integrity $[2,31]$.

It activates antioxidants, including vitamin $C$, glutathione and vitamin $\mathrm{E}$. Vitamin $\mathrm{C}$ is an interesting nutrient that functions in the prevention and reduction of the severity of viral diseases due to its various physiological properties such as trapping free radicals, reducing gene expression of proinflammatory cytokines, and its germicidal effect on some cell types [31,32]. It regulates the proliferation and function of neutrophils and monocytes, and enables neutrophil migration to the infection site [33]. Vitamin C protects body cells and tissues against oxidative damage and dysfunction while scavenging harmful reactive oxygen species [34]. It protects DNA, proteins and lipids against oxidative damage by capturing free radicals [13]. In a study conducted on the use of vitamin $C$ in the treatment of Covid-19 epidemic, it has seen that thanks to its antioxidant activity, it reduced oxidative stress inflammation, increased immune cell function [35].

The results of five studies involving 471 patients requiring more than 10 hours of ventilation were found to reduce the duration of ventilation by an average of $25 \%$ with vitamin C supplementation of 1-6g / day [36]. With its powerful antioxidant effect, vitamin $\mathrm{C}$ can be used to reduce oxidative stress caused by cytokine storm; It has been suggested that intravenously high doses of vitamin C (10-20 $\mathrm{g} /$ day) may have positive effects in individuals with severe Covid-19 [37].

\section{Thiamine (Vitamin B1)}

Thiamine (Vitamin B1) plays a key role in metabolic systems as a coenzyme in energy metabolism, mainly in the form of thiamine pyrophosphate (TPP), particularly carbohydrate and protein metabolism [38]. Thiamine activates the immune system and has an anti-inflammatory effect $[39,40]$. It is predicted that thiamine deficiency may increase the development of ARDS due to Covid-19 [24].

\section{Riboflavin (Vitamin B2)}

Vitamin B2 combines with phosphoric acid in tissues and exists as flavin mononucleotide (FMN) and flavin adenine dinucleotide (FAD). It has been suggested that riboflavin may play a role in regulating the immune inflammation associated with the Covid-19 epidemic with its effects on reducing cytokine release [24].

\section{Niacin (Nicotinic Acid, Vitamin B3)}

Niacin functions as a coenzyme in the body in the form of nicotinamide adenine dinucleotide (NAD), nicotinamide adenine dinucleotide phosphate (NADP) [41]. Studies have shown that Niacin was highly effective in preventing lung tissue damage and this micronutrient supplement was infected with Covid-19. It has been reported that its application to patients who have been diagnosed may be a correct approach [42]. It has been stated that niacin, with its immune regulatory effect, may play a role in the prevention of inflammatory complications associated with Covid-19 infection [24].

\section{Folate / Folic Acid (Vitamin B9)}

Folic acid is important for antibody production and metabolism. It has been reported to improve immune function and protect against infections [43]. Folic acid can stop the entry of Covid-19 into the cell by binding the furin enzyme with strong intermolecular interaction. It has been reported that with this effect, folic acid can help prevent or alleviate respiratory involvement associated with Covid-19 [24].

\section{Cobalamin (Vitamin B12)}

Vitamin B12 functions as a modulator of human immunity by facilitating the production of T-lymphocytes recruited in cellular immunity, increasing immune activation against viruses and bacteria, and increasing the number of natural killer cells [44]. Cobalamin is vital for erythrocyte production and DNA synthesis. In the study, it was stated that vitamin B12 can reduce the severity of the Covid-19 outbreak by inhibiting the RNA-dependent RNA polymerase enzyme responsible for the replication of the viral genome [45].

\section{Iron}

Iron, the most abundant trace element in the human body; It plays a key role in ATP production, oxygen transport, DNA and protein synthesis, erythrocyte production, electron transport, cellular respiration, cell proliferation, and regulation of gene expression [46]. There are several studies suggesting that iron chelates can be used as supportive therapy in the treatment of Covid-19, a virus that requires 


\section{International Journal of Biochemistry \& Physiology}

iron [47]. It has been reported that the optimum level of iron intake with diet and the absence of deficiency or overload may be effective in preventing immune system complications caused by Covid-19 [24].

\section{Zinc}

Zinc is a trace element that has a key role in the development and function of both natural and acquired immunity $[11,48]$. It has been shown to reduce replication in a wide variety of RNA viruses, including coronavirus, in in vitro studies [33,49-51]. In one study, it was reported that zinc inhibits the binding and elongation of the coronavirus RNA-dependent RNA polymerase (RdRp) template [25]. It has been stated that Covid-19 will affect host resistance against viral infection by minimizing the disease burden and may create an additional shield against Covid-19 pandemic [52]. Chloroquine (CQ) and hydroxychloroquine (HCQ) used in the treatment have a significant inhibitory effect on intracellular $\mathrm{pH}$ increase. It has been observed that zinc given in addition to chloroquine and hydroxychloroquine affects a rapid improvement in the course of the disease [53,54]. Zinc is required for the normal function and development of nonspecific immune-regulating cells, including natural killer cells and neutrophils [11].

\section{Selenium}

Selenium is an essential element that plays a role in many mechanisms in the body, including thyroid hormone metabolism, antioxidant defense and regulation of the immune system, and participates in many enzymes as a cofactor [55]. In a study conducted in China, it was emphasized that there is a relationship between selenium levels detected from hair samples and Covid-19 healing rates and that the effectiveness of selenium Covid-19 is worth investigating [12]. Selenoproteins have been reported to protect against oxidative stress induced by reactive oxygen species (ROS) and reactive nitrogen species (NOS) with their antioxidant effects [56]. Selenium has been reported to have clinical benefits in RNA viral infections [57]. When Covid-19 cases are examined, it is thought that especially the elderly population is at risk of selenium deficiency and therefore they may be vulnerable to Covid-19 [12].

\section{Omega-3 Polyunsaturated Fatty Acids}

Omega-3 polyunsaturated fatty acids have been reported to have anti-inflammatory properties and play an important role in relieving inflammation. It has been reported that omega-3 supplementation has positive effects on Acute Respiratory Distress Syndrome (ARDS), thereby alleviating adverse effects associated with Covid-19 infection [24,58].

\section{Pre-Probiotics}

Probiotics are defined as living microorganisms that, when administered in sufficient quantities, provide a health benefit to the host, including the gastrointestinal tract. Lactobacillus and Bifidobacterium species have been found to be decreased in Covid-19 patients $[8,59,60]$. In a study, it was reported that probiotic supplementation reduced the risk of infection or shortened its duration [2]. In a study conducted in China, Covid-19 was shown to cause dysbiosis (deterioration in the microbiota) [60]. Covid-19 virus uses ACE2 receptors, which are also commonly found in the intestines, to enter cells. These receptors provide transport of neutral amino acids, antimicrobial proteins and play a role in maintaining the balance of intestinal microbiota. Studies have shown that intestinal dysbiosis disrupts the balance between the Renin-Angiotensin-Aldosterone system (the hormonal system that regulates blood pressure and fluid balance), ACE and ACE2 axes in the kidney, the increase in ACE2 receptors accelerates the development of diabetic nephropathy and kidney damage increases rapidly. While Covid-19 was initially thought to be transmitted only by droplets, it was also transmitted via fecal oral (fecal) route. The ayes have it. It has been stated that targeting balanced bacterial microbiota may be a part of preventive therapy and therapy in order to prevent the passage of the virus, which can attach to the ACE 2 receptor in the intestinal mucosa, to the intestinal mucosa via the stool [60].

\section{Conclusion}

Considered a pandemic, the Covid-19 outbreak poses an important threat to the whole world. Potential therapeutics and prevention strategies that can reduce the severity of the disease are vital, as there are currently no drugs or other therapeutic agents approved for the treatment of Covid-19.

The immune system is the body's natural defense mechanism. When it works properly, it prevents many diseases, improves the symptoms of the diseases and shortens the duration. The most important factor in strengthening immunity and protecting health; It is an optimal diet that is sufficient and balanced in terms of macronutrients and rich in micronutrients.

Thanks to experimental research, studies with people with macro and micronutrient deficiencies, a number of vitamins (A, C, and E), omega-3 fatty acids, pre / probiotics and trace elements (zinc, selenium) to support the human immune system and reduce the risk of infection. Vitamin A has an immunity-enhancing effect. Because of its functions in the epithelial tissue, it becomes easier for infections to enter the body when it is insufficient. In addition, considering the effects of Covid-19 on lung function, vitamin A deficiency is especially important due to changes in the lung epithelium. Oral vitamin C supplementation can be protective in risky groups to prevent viral infections such as Covid-19. In addition, intravenous vitamin $\mathrm{C}$ administration appears 


\section{International Journal of Biochemistry \& Physiology}

to be a safe and inexpensive method of treatment with better results. Vitamin $\mathrm{E}$ is effective in regulating immune functions. Increasing vitamin $\mathrm{E}$ intake can strengthen the immune system. As a result; For a more functional and active immune system, healthy, adequate and balanced nutrition will decrease the mortality of individuals and increase the quality of life.

\section{References}

1. Cao X (2020) COVID-19: immunopathology and its implications for therapy. Nature Reviews Immunology 20: $269-270$

2. Jayawardena $\mathrm{R}$, Sooriyaarachchi $\mathrm{P}$, Chourdakis $\mathrm{M}$, Jeewandara C, Ranasinghe P (2020) Enhancing immunity in viral infections, with special emphasis on COVID-19: A review Diabetes \& Metabolic Syndrome: Clinical Research \& Reviews 14(4): 362-382.

3. Iron $\mathrm{H}$, Evliyaoğlu $\mathrm{K}$, Aka $\mathrm{N}$, The Importance of biochemical parameters in the Coronavirus Disease 2019 Covidien 19v Centennial University, Faculty of Science, Department of Chemistry, Biochemistry Department, 65100, Van-Turkey.

4. Guo YR, Cao QD, Hong ZS, Tan YY, Chen SD, et al. (2020) The origin, transmission and clinical therapies on coronavirus disease 2019 (COVID-19) outbreak - an update on the status. Mil Med Res 7: 11.

5. Song Z, Xu Y, Bao L, Zhang L, Yu P, et al. (2019) From SARS to MERS, thrusting corona-viruses into the spotlight. Viruses 11(1): 59.

6. Zhou Y, Fu B, Zheng X, Wang D, Zhao C, et al. (2020) Aberrant pathogenic GM-CSF + T cells and inflammatory CD14 + CD16 + monocytes in severe pulmonary syndrome pa-tients of a new coronavirus. bioRxiv 2020 .

7. Mehta P, McAuley DF, Brown M, Sanchez E, Tattersall RS, et al. (2020) COVID-19: con-sider cytokine storm syndromes and immunosuppression. Lancet 395(10229): 1033-1034.

8. Xu X, Han M, Li T, Sun W, Wang D, et al. Effective treatment of severe COVID-19 patients withtocilizumab. Proc Natl Acad Sci U S A 117(20): 10970-10975.

9. Karaağaç Y, Koyu EB (2020) Vitamins and Minerals in Viral Infections: A Review Focused on COVID-19. İzmir Kâtip Çelebi University Faculty of Health Sciences Journal 5(2): 165-173.

10. Sarıtaş A, Çalışkan T, Öztop MB (2020) Nutrition in SARS-CoV-2 (Covid-19) Disease. Tepecik Eğit Hast Derg
30(2): 118-125.

11. Gasmi A, Noor S, Tippairote T, Dadar M, Menzel A (2020) Individual risk management strategy and potential therapeutic options for the COVID-19 pandemic. Clnical Immunology 215: 108409.

12. Zhang J, Taylor EW, Bennett K, Saad R, Rayman M (2020) Association Between Regional Selenium Status and Reported Outcome of COVID-19 Cases in China. Am J Clin Nutr 111: 1297-1299.

13. Bakan S, Deveboynu ȘN, Kartal FT (2020) The Effect of Antioxidant Vitamins on Immunity in the COVID-19 Pandemic. Eurasian JHS 3(COVID-19 Special Issue): 140148.

14. Singh RP, Sharad S, Kapur S (2004) Free radicals and oxidative stress neurodegenerative diseases: relevance of dietary antioxidants. Journal Indian Academy of Clinical Medicine 5(3): 218-225.

15. Ekici L, Sağdıç O (2008) Inhibition of Free Radicals and Antioxidant Foods Erciyes University, Faculty of Engineering, Department of Food Engineering, Kayseri Gida 33(5): 251-260.

16. Çavdar C, Sifil A, Çamsarı T (1997) Reactive Oxygen Particles and Antioxidant Defense. Office Journal of the Turkish Nephrology, Association 6(2): 92-95.

17. Vinson JA (2006) Oxidative stress in cataracts. Pathophysiology 13(3): 151-162

18. Velioğlu S (2000) The Effects of Natural Antioxidants on Human Health. Food 25: 167-176.

19. Kasnak C, Palamutoğlu R (2015) Classification of Natural Antioxidants and Their Effects on Human Health Turkish Agriculture. Food Science and Technology Journal 3(5): 226-234.

20. Ross SA, McCafferry PJ, Drager UC, Luca LM (2000) Retinoids in Embryonal Development. Physiological Reviews 80(3): 1022-1046.

21. Khaled MB, Benajiba N (2020) The role of nutrition in strengthening immune system against newly emerging viral diseases: case of SARS-CoV-2. Nor Afr J Food Nutr Res 4(7): 240-284.

22. Dowling JE (2020) Vitamin A: Its many roles-from vision and synaptic plasticity to infant mortality. J Comp Physiol A Neuroethol Sens Neural Behav Physiol 206(3): 389-399.

23. Semba RD (1999) Vitamin A and immunity to viral, 


\section{International Journal of Biochemistry \& Physiology}

bacterial and protozoan infections. Proc Nutr Soc 58(3): 719-727.

24. Tek N.A., KoçakT (2020) The Role of Nutrition in Supporting the Immune System in Combating Coronavirus (covid-19). Gazi Journal of Health Sciences, pp; 18-45.

25. Topuz HŞ (2020) Nutrition in Covid-19 Infection Med Res Rep 3(1): 176-180.

26. Lewis DE, Meydani NS, Wu D (2019) Regulatory role of vitamin $\mathrm{E}$ in the immune system and inflammation. IUBMB Life 71(4): 487-494.

27. Meydani SN, Han SN, Wu D (2005) Vitamin E and immune response in the aged: molecular mechanisms and clinical implications. Immunological reviews 205(1): 269-284.

28. Stipp MM (2020) SARS-CoV-2: Micronutrient optimization in supporting host immunocompetence. International Journal of Clinical Case Reports and Reviews 2(2): 1-11.

29. Lewis DE, Meydani NS, Wu D (2019) Regulatory role of vitamin $\mathrm{E}$ in the immune system and inflammation. IUBMB Life 71(4): 487-494.

30. Calder CP (2020) Nutrition, immunity and COVID-19. BMJ Nutrition, Prevention \& Health 2020; 0.

31. Adams K K, Baker L W, Sobieraj M D. Myth busters: Dietary supplements and COVID-19. Annals of Pharmacotherapy 2020: 1-7.

32. Gombart AF, Pierre A, Maggini S (2020) A review of micronutrients and the immune system - working in harmony to reduce the risk of infection. Nutrients 12(1): 236.

33. Calder PC, Carr AC, Gombart AF, Eggersdorfer M (2020) OptimalNutritional Status for a WellFunctioning Immune System Is an Important Factor to Protect against Viral Infections. Nutrients 12(1): 236.

34. Carr AC, Maggini S (2017) Vitamin C and immune function. Nutrients 9(11): 1211.

35. Rosa SGV, Santos WC. Clinical trials on drug repositioning for COVID-19 treatment. Revista Panamericana de Salud Pública 44: e40..

36. Hemilia H, Chalker E (2020) Vitamin C may reduce the duration of mechanical ventilation in critically ill patients: a meta-regression analysis. Journal of Intensive Care 8(1): 15.
37. Cheng RZ (2020) Can early and high intravenous dose of vitamin C prevent and treat coronavirus disease 2019 (COVID-19)? Medicine in Drug Discovery 5: 100028.

38. Manzetti S, Zhang J, van der Spoel D (2014) Thiamin function, metabolism, uptake, and transport. Biochemistry 53(5): 821-835.

39. Kunisawa J (2017) Metabolic changes during B cell differentiation for the production of intestinal IgA antibody. Cellular and Molecular Life Sciences 74(8): 1503-1509.

40. Spinas E, Saggini A, Kritas S, Cerulli G, Caraffa A, et al. (2015) Crosstalk between vitamin B and immunity. J Biol Regul Homeost Agents 29(2): 283-288.

41. Canto C, Menzies KJ, Auwerx J (2015) NAD + metabolism and the control of energy homeostasis: a balancing act between mitochondria and the nucleus. Cell metabolism 22(1): 31-53.

42. Shi Y, Wang Y, Shao C, Huang J, Gan J, Huang X, et al. COVID-19 infection: the perspectives on immune responses. Cell Death \& Differentiation volume 27: 1451-1454.

43. Troen AM, Mitchell B, Sorensen B, Wener MH, Johnston A, et al. (2006) Unmetabolized folic acid in plasma is associated with reduced natural killer cell cytotoxicity among postmenopausal women. The Journal of nutrition. 136(1): 189-194.

44. Saeed F, Nadeem M, Ahmed RS, Tahir Nadeem M, Arshad MS, et al. (2016) Studying the impact of nutritional immunology underlying the modulation of immune responses by nutritional compounds - a review. Food and Agricultural Immunology 27(2): 205-229.

45. Narayanan N, Nair DT. Vitamin B12 May Inhibit RNADependent-RNA Polymerase Activity of nsp12 from the COVID-19 Virus. 2020.

46. Puig S, Ramos-Alonso L, Romero AM, Martínez-Pastor MT (2017) The elemental role of iron in DNA synthesis and repair. Metallomics 9(11): 1483-500.

47. Dalamaga M, Karampela I, Mantzoros CS (2020) Commentary: Could iron chelators prove to be useful as an adjunct to COVID-19 treatment regimens? Metabolism Clinical and Experimental 108: 154260.

48. Maares M, Haase H (2016) Zinc and immunity: an essential interrelation. Arch Biochem Biophys 611: $58-65$. 


\section{International Journal of Biochemistry \& Physiology}

49. Read SA, Obeid S, Ahlenstiel C (2019) The role of zinc in antiviral immunity. Adv Nutr 10(4): 696-710.

50. Derwand R, Scholz M (2020) Does zinc supplementation enhance the clinical efficacy of chloroquine / hydroxychloroquine to win today's battle against COVID-19? Medical Hypotheses 142: 109815.

51. Velthuis AJW, van den Worm SHE, Sims AC, Baric RS, Snijder EJ, et al. (2010) Zn (2+) inhibits coronavirus and arterivirus RNA polymerase activity in vitro and zinc ionophores block the replication of these viruses in cell culture. PLOS Pathog 6(11): e1001176.

52. Razzaque M (2020) COVID-19Pandemic: Can Maintaining Optimal Zinc Balance Enhance Host Resistance? 251(3): 175-181.

53. Skalny AV, Rink L, Ajsuvakova OP, Aschner M, Gritsenko VA, et al. (2020) Zinc and respiratory tract infections: Perspectives for COVID19 (Review). International Journal of Molecular Medicine 46(1): 17-26.

54. Shittu MO, Afolami OI (2020) Improving the efficacy of chloroquine and hydroxychloroquine against SARSCoV-2 may require zinc additives - A better synergy for future COVID-19 clinical trials. Infez Med 28(2): 192197.
55. Kangalgil M, Assistant $H$ (2017) The effects of selenium on human health and its relationship with diabetes mellitus. Bozok Medical Journal 7(4): 66-71.

56. Tinggi U (2008) Selenium: its role as antioxidant in human health. Environmental health and preventive medicine 13(2): 102-108.

57. Güngör EÖ, Yaldız N, Özbek SÇ (2020) Micronutrients If You Support the Immune System: A Review for COVIT 19 YIU Health Science Deg 1: 53-56.

58. Li C, Bo L, Liu W, Lu X, Jin F (2015) Enteral Immunomodulatory Diet (Omega-3 Fatty Acid, $\gamma$-Linolenic Acid and Antioxidant Supplementation) for Acute Lung Injury and Acute Respiratory Distress Syndrome: AnUpdated Systematic Review and MetaAnalysis. Nutrients 7(7): 5572-5585.

59. Mak J, Chan F, Ng SC (2020) Probiotics and COVID-19: one size does notfit all. The lancet. Gastroenterology \& hepatology 5(7): 644-645.

60. Acarkan T, Erdoğan D, Kaçar M (2020) The Role of Lung and Gut Microbiota in The Combat Against COVID-19. Anatolian Clinic Medical Sciences Journal 25(S1): 284293. 